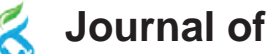 Chemistry \& Applications
}

\section{The Effect of Parylene-C Coating on the Surface Free Energy, Water Sorption, Solubility and Staining of PMMA}

Keywords: Parylene; PMMA; Surface free energy; Water sorption; Water solubility; Sta ining; Coating

\begin{abstract}
Background and objective: Parylenes are conformal protective polymer coating materials utilized in various biomedical applic ations since they are chemically and biologically inert and stable. Acrylic resins, used in the manufacturing of different types of intra-oral prostheses, are vulnerable to fluid sorption and solubility and consequently alteration of different physical and mechanical properties. This study investigated the effect of surface coating of polymethyl metha crylate (PMMA) with Parylene-C, on surface free energy, water sorption, solubility, and staining.
\end{abstract}

Methods: Specimens were fabricated using heat-polymerizing PMMA, half were randomly chosen to be coated with Parylene-C and various groups were created for testing as follows: 24 specimens were subjected to water sorption and solubility tests in distilled water according to ISO 20795-1:2008 fordenture base polymers. 48 spec imens underwent staining tests by soaking in distilled water or coffee solution for one week. Discoloration was measured by companing total colour differences $(\triangle E)$ and lightness values $\left(L^{*}\right)$ across groups after obtaining CIE L*a*b* values of all samples using a digital camera imaging and appropriate image analysis software. A further 40 samples were subjected to contact angle readings using deionized water and glycerol followed by surface free energy calculations. Nonparametric Mann-Whitney tests were used for sta tistic al a na lysis $(\mathrm{P} \varangle 0.01)$.

Results: Coated PMMA specimens demonstrated significantly less water somtion and lower surface free energy compared to noncoated $(P<0.001)$, while no significant difference was found in solubility $(P=.028)$. The coating did not have a significant effect on $\Delta E$ values after the staining tests, but the $L^{*}$ values in coated samples were significantly higher compared to the uncoated ones.

Significance: This study forms part of a series of experiments on the effect of Parylene coating of PMMA. The modified surface properties of coated PMMA may prolong the lifetime of intraoral prostheses and possibly reduce biofilm formation.

\section{Introduction}

Poly-methyl methacrylate (PMMA) resin is a low cost material that is easy to handle and has been used in dentistry for many years for the fabrication of intraoral prostheses. However, it is not an ideal material in every aspect [1]. It is subject to sorption, a process of adsorption and absorption of liquids dependent on the environmental conditions [2]. Should a contacting solution be pigmented, discoloration is also possible during service in the oral environment [2]. The sorption phenomenon is of considerable importance since it results in dimensional changes [3,4], as well as a reduction of flexural strength [5,6], impact strength [7], transverse strength [8] and fatigue life of PMMA [9]. Another problem of PMMA is the presence of surface and subsurface voids which may compromise its

\section{Fariba Motevasselian ${ }^{1}$, Eugene Cheung ${ }^{1}$, Steven} $\mathrm{Soo}^{1}$, Aviva Petrie ${ }^{2}$ and Haralampos Petridis ${ }^{1 *}$

${ }^{1}$ Department of Restorative Dentistry, Prosthodontics Unit, UCL Eastman Dental Institute, London, UK

${ }^{2}$ Biostatistics Unit, UCL Eastman Dental Institute, London, UK

\section{Address for Correspondence}

Haralampos Petridis, Department of Restorative Dentistry, Prosthodontics Unit, UCL Eastman Dental Institute, 256 Grays Inn Road, London, WC1X 8LD, Tel: +44(0) 203456 1250; E-mail: c.petridis@ucl.ac.uk

Copyright: $\odot 2014$ Motevasselian F, et al. This is an open access article distributed under the Creative Commons Attribution License, which permits unrestricted use, distribution, and reproduction in any medium, provided the original work is properly cited.

Submission: 25 April 2014

Accepted: 10 June 2014

Published: 13 June 2014

physical, aesthetic and hygiene properties [10]. These surface defects and porosities provide favourable niches for the development and differentiation of microbial colonies [11]. In addition, rough PMMA surfaces may predispose to colour changes [12]. Resin-based dental materials are prone to discoloration $[1,2,13]$ and colour changes after exposure to denture cleansers and beverages $[14,15]$.

Parylenes is a generic name of a unique polymer series which are widely used in medical sciences as a coating material. There are several variations of Parylene based on its monomer derivatives [16]. Parylene- $\mathrm{C}$ is a thermoplastic, crystalline and transparent polymer that is extensively used as a coating for insulating implantable biomedical devices [17]. In addition, Parylene- $\mathrm{C}$ is chemically inert and non-biodegradable [18]. Parylene-C is synthesized from a low molecular weight (MW) dimer, dichloro-di (p-xylylene), using a process that involves the decomposition of $\mathrm{p}$-xylylene to yield chlorop-xylylene, followed by the polymerization of chloro-p-xylylene to Parylene-C [19]. Parylene-C can be vapour deposited onto substrates to generate uniform, pinhole free and hydrophobic membranes [20]. Parylenes may be used for the coating of intraoral prostheses made of PMMA, in order to reduce microbial colonization and improve surface properties [21]. The purpose of the present study was to investigate the effect of surface coating of PMMA with Parylene-C on water sorption, solubility, surface free energy, and staining.

\section{Material and Methods}

A commonly used heat polymerizing PMMA resin (Diamond D ultra-impact denture acrylic, Keystone Industries, New Jersey, USA) was used in the present study. A large metal two-part flask was used to prepare a stone mould for packing of the heat-cured acrylic resin dough to produce a sheet of PMMA resin with a thickness of $1.5 \mathrm{~mm}$. The PMMA was processed under a slow cycle ( 8 hours) according to the manufacturer's recommendations. This was then sectioned to produce 24 square specimens of $50 \mathrm{~mm}$ side for the water sorption and solubility tests, 48 square specimens of $15 \mathrm{~mm}$ for the staining test, and 40 square specimens of $15 \mathrm{~mm}$ for the surface free energy test. All the specimens were finished by hand by using progressively finer grades of silicon carbide water-proof paper sheets $(240,320$, 400 and 600 grit) until an even unblemished surface was produced similar to that encountered when making an intra-oral prosthesis. 
Citation: Motevasselian F, Cheung E, Soo S, Petrie A, Petridis H. The Effect of Parylene-C Coating on the Surface Free Energy, Water Sorption, Solubility and Staining of PMMA. J Chem Applications. 2014;1(1): 7.

The specimens were coded with a small round bur on the periphery of one side, steam cleaned for 5 seconds and dried with tissue paper. Half of all the specimens were coated with Parylene-C to a thickness of $10 \mu \mathrm{m}$ (Specialty Coating Systems Ltd, Surrey, UK). Before coating, the specimens were cleaned by rinsing with de-ionised water and 2-iso-propanol (IPA), and treated with the company's chemical adhesion promoter (Adpro Plus, SCS Ltd, Surrey, UK). The samples were fixed by use of a crocodile clip in one corner. The parts were coated to a thickness of $5 \mu \mathrm{m}$, then the crocodile clip was moved to a new position before continuing coating to a total of 10 microns. The contact point created by the first position of the crocodile clip would therefore be the only point coated to $5 \mu \mathrm{m}$. Control coupons were also placed within the chamber in order to verify the final thickness. The whole coating process was performed by the respective company (Specialty Coating Systems Ltd, Surrey, UK) without disclosing details of the process.

\section{Water sorption and solubility test}

Twelve uncoated and 12 Parylene-C coated PMMA samples were tested following the recommendations of ISO 20795-1:2008 for base polymers [22]. The specimens were placed vertically on a wire rack to avoid sample to sample contact. The specimens were first conditioned to a constant mass by placing them in desiccators containing thoroughly dry anhydrous silica gel (Silica gel desiccant, Fisher Scientific, New Jersey, USA). The desiccators were placed in an incubator (LTE Raven, Richmond Scientific Ltd., Lancashire, UK) at $37^{\circ} \mathrm{C}$ for 24 hours. Following removal from the desiccators, each specimen was weighed on a scale (AG204 balance, Mettler, Toledo, Switzerland) with a precision of $0.1 \mathrm{mg}$. This drying cycle was repeated until the weight loss of each specimen was no greater than $0.2 \mathrm{mg}$ in any 24 hour period. At this point, the weight was considered constant (W1).

Next, the specimens were immersed in distilled water at $37^{\circ} \mathrm{C}$ for seven days. The water was changed every day to prevent saturation by soluble components. They were removed from the water with tweezers and wiped with a clean tissue paper until free from visible moisture and weighed again (W2). The specimens were finally reconditioned to a constant mass (W3) following the same protocol as for conditioning the specimens prior to water immersion. The final values of water sorption and solubility were obtained using the following formulas [22]:

Water sorption $(\mathrm{Wsp})=\mathrm{W} 2(\mu \mathrm{g})-\mathrm{W} 3(\mu \mathrm{g}) /$ sample volume $\left(\mathrm{mm}^{3}\right)$

Water solubility $(\mathrm{Wsl})=\mathrm{W} 1(\mu \mathrm{g})-\mathrm{W} 3(\mu \mathrm{g}) /$ sample volume $\left(\mathrm{mm}^{3}\right)$

\section{Surface free energy test}

All 40 samples were subjected to contact angle readings using deionized water and glycerol followed by surface free energy calculations. A goniometer (Cam 200, KSV Instruments Ltd., Helsinki, Finland) was used for contact angle measurements. A droplet of approximately $10 \mu \mathrm{l}$ was allowed to drop onto the sample after the software was preprogrammed. This allowed frames to be captured at 40 millisecond intervals until one second had been reached ( 25 frames). The solid/ liquid/gas intersect could be determined using software analysis of the drop profile and multiple contact angles measured from the images.
Both the left and right contact angles of the droplet were measured on each frame allowing for 50 measurements to be included for the mean value calculations. The protocol was repeated until measurements on all 40 samples were made using the two different liquids. Appropriate software (SFECam2008, KSV Instruments Ltd., Helsinki, Finland) was used to calculate the surface free energy of the samples using the surface tension data of the two reference liquids, mean contact angle data, and the Owens-Wendt theory [23].

\section{Staining test}

48 square $(15 \mathrm{~mm})$ PMMA specimens were used for the staining test. Half were left uncoated and half were coated with Parylene-C, in a random manner. Half of the specimens $(\mathrm{N}=12)$ in each group were immersed in distilled water at $40^{\circ} \mathrm{C}$ and half were immersed in a coffee solution made using $40 \mathrm{~g}$ of coffee powder (Gold Blend, Nestle Ltd, Gatwick, UK) dissolved in one litre of boiling distilled water. The coffee solution was stirred at $250 \mathrm{rpm}$ on a magnetic hotplate/stirrer (Model MR 3002 S, Heidolph, UK) to prevent settling of any coffee powder. All immersed specimens were placed in an incubator at $40^{\circ} \mathrm{C}$ for one week (LTE Raven, Richmond Scientific Ltd., UK). At the end of the test period, the specimens were removed from the solutions and rinsed in distilled water. Excess water on the surfaces was removed with tissue paper and the specimens were allowed to dry.

A high resolution, 18 mega-pixel, digital camera (EOS 550D, Canon, Tokyo, Japan) with a $100 \mathrm{~mm}$ macro lens (Canon, Tokyo, Japan) was used to obtain photographs of the specimens. The settings used were: aperture set to f5.6, shutter speed to $1 / 125 \mathrm{~s}$ and ISO was set at 400 . Every effort was made in order to make the exposure parameters constant and uniform throughout the experiment: The white balance was set to tungsten light with a colour temperature of $3200 \mathrm{~K}$ which was the closest setting to the actual colour temperature of the light source $(3400 \mathrm{~K})$. The camera was mounted on a rigid adjustable copy stand at $41 \mathrm{~cm}$ distance from camera to specimen. The distance between the front end of the macro lens and the specimen was $16.5 \mathrm{~cm}$. The light source (KL1500-T, Schott, Stafford, UK) consisted of one halogen lamp (15 Voltage, 150 watt, $3400 \mathrm{~K}$ ) with two flexible fibre-optic arms. These were set at $45^{\circ}$ to the axis of the lens by means of a plastic holder because the diffuse reflection responsible for colour occurs at $45^{\circ}$ from the incident light [24] (Figure 1). The lens was focused manually on the sample. Each specimen, to be measured was placed on a grey card (Art Shop, UK) ensuring the specimen was in the middle of the view finder. A middle-gray background was used as a way to produce consistent image exposure and colour in film photography [25]. Images were saved as high quality and then analysed using imaging software (Adobe Photoshop CS4 software Adobe, San Jose, USA) enabling the collection of quantitative colour parameters ( $\mathrm{L}$, a, and $\mathrm{b}$ values) $[24,26]$. The image was outlined using the marquee tool within the toolbar option of Photoshop, to select the maximum area of the specimen while avoiding the borders. A histogram was then provided that gave the mean $\mathrm{L}$, $\mathrm{a}$, and $\mathrm{b}$ values of all pixels within the selected area. The lightness, $a$, and $b$ values in the histogram window are not standard colour values. However, they can be converted to $L^{*}, a^{*}, b^{*}$ values of CIE LAB using the following formulas [27]:

$$
\begin{aligned}
& \mathrm{L}^{*}=\text { Lightness } / 255 \times 100 \\
& \mathrm{a}^{*}=240 \mathrm{a} / 255-120
\end{aligned}
$$


Citation: Motevasselian F, Cheung E, Soo S, Petrie A, Petridis H. The Effect of Parylene-C Coating on the Surface Free Energy, Water Sorption, Solubility and Staining of PMMA. J Chem Applications. 2014;1(1): 7.

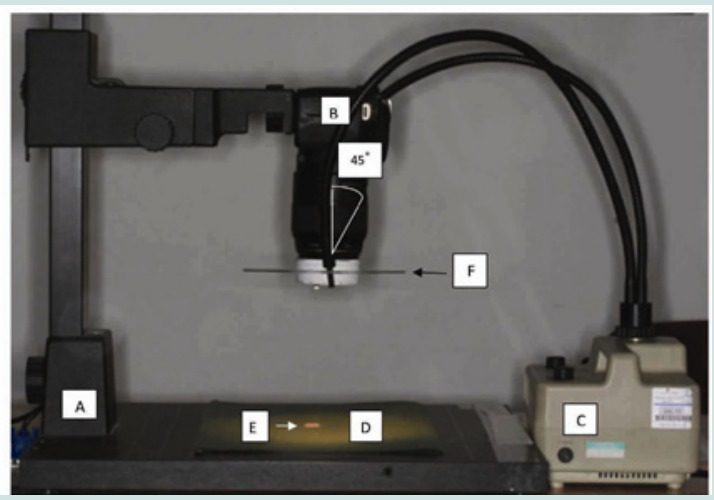

Figure 1: The digital image capture set-up. (A: rigid adjustable copy stand. B: digital camera. C: light source. D: middle-gray background. E: a specimen. F: Plastic holder).

\section{$\mathrm{b}^{*}=240 \mathrm{~b} / 255-120$}

The CIE system colour values ${ }^{28}\left(\mathrm{~L}^{*}, \mathrm{a}^{*}\right.$ and $\left.\mathrm{b}^{*}\right)$ of all the specimens were analysed before and after exposure to any medium. Colour differences $(\Delta \mathrm{E})$ of the specimens were calculated according to the equation ${ }^{28}: \Delta \mathrm{E}^{*} \mathrm{ab}=\left[\left(\Delta \mathrm{L}^{*}\right)^{2}+\left(\Delta \mathrm{a}^{*}\right)^{2}+\left(\Delta \mathrm{b}^{*}\right)^{2}\right]^{1 / 2}$

Where, $\Delta \mathrm{L}, \Delta \mathrm{a}$, and $\Delta \mathrm{b}$ are differences in $\mathrm{L}$, $\mathrm{a}$, and $\mathrm{b}$ values before and after immersion in a solution. The reproducibility of the imaging system was tested by taking images of dental composite resin shade tabs (Gradia, GC Co, Tokyo, Japan). Three photographs were taken on three different days, of the middle portion of the shade tabs A1 and A4 and the relevant CIE $L^{\star} a^{*} b^{\star}$ values were obtained.

\section{Scanning electron microscopy}

Several specimens were prepared for Scanning Electron Microscopy (SEM) analysis by sputter coating with gold/palladium. A scanning electron microscope (JSM-5410 L, Jeol, Tokyo, Japan) operating at $10 \mathrm{KV}$ was used at $\mathrm{x} 1000$ and X3500 magnification on the surface the specimens.

\section{Statistical analysis}

Non-parametric Mann-Whitney tests were used to compare the distributions of water sorption, solubility, surface free energy, and colour difference $(\Delta \mathrm{E})$ in PMMA coated and non-coated specimens after one week's immersion in each of two media as the data were not normally distributed. The Mann Whitney tests were also used to compare the $\Delta \mathrm{E}$ after immersion in distilled water and coffee for both coated and uncoated PMMA specimens, and to compare the lightness of the specimens in the two groups before and after immersion in each medium. The non-parametric Wilcoxon signed rank test was used to assess the effect of the staining test on the lightness shift of the specimens after immersing them either in distilled water or coffee. A significance level of 0.01 was chosen rather than the conventional 0.05 to avoid falsely significant results arising from multiple testing. Appropriate software was used for statistical analysis (SPSS version 20, IBM, Portsmouth, UK)

\section{Results}

\section{Water sorption and solubility}

The median, minimum and maximum values of water sorption and solubility are presented in Table 1. On average, there was significantly less water sorption in Parylene-C coated PMMA than in non-coated PMMA specimens $(\mathrm{P}<0.001)$, while no significant difference was found in the solubility between the groups $(\mathrm{P}=.028)$.

\section{Surface free energy}

Table 2 shows the descriptive statistics calculated for the two sample groups. There was a statistically significant difference ( $\mathrm{p}<$ 0.001) in the median surface free energy values between the two groups, with the median value for the uncoated PMMA group being consistently higher than the Parylene coated group $\left(38.36 \mathrm{~mJ} / \mathrm{m}^{2}\right.$ compared to $14.52 \mathrm{~mJ} / \mathrm{m}^{2}$ ).

The interquartile range also differed between the two groups with the coated group presenting with a smaller range and more consistent values (Table 2).

\section{Staining test}

The median, minimum and maximum of colour difference $\Delta \mathrm{E}$ of PMMA and Parylene-C coated PMMA specimens after one week immersion in distilled water or coffee solution are presented in Table 3. There was no statistically significant difference in the distributions when the $\triangle \mathrm{E}$ values of coated vs. uncoated PMMA samples were compared after immersion in distilled water or in coffee solution for one week ( $\mathrm{P}=0.52$ and 0.932 respectively). The storage medium also did not exhibit any statistically significant effect when the $\Delta \mathrm{E}$ values after immersion for uncoated and coated sample groups were compared ( $\mathrm{P}=0.128$ and 0.834 , respectively).

Furthermore, the lightness values of all groups of samples,

Table 1: Water sorption and water solubility of the test materials.

\begin{tabular}{|c|c|c|c|}
\hline \multicolumn{1}{|l|}{ Group } & Wsp $\left(\mu \mathrm{g} / \mathbf{m m}^{3}\right)$ & Wsl $\left(\mu \mathbf{g} / \mathbf{m m}^{3}\right)$ \\
\hline \multirow{3}{*}{ PMMA } & $\mathrm{N}$ & 12 & 12 \\
\cline { 2 - 4 } & Median & 26.41 & 1.06 \\
& Minimum & 17.67 & .92 \\
& Maximum & 28.20 & 1.18 \\
\hline \multirow{3}{*}{ Parylene-coated } & $\mathrm{N}$ & 12 & 12 \\
\cline { 2 - 4 } PMMA & Median & 15.32 & .97 \\
& Minimum & 14.89 & .52 \\
& Maximum & 16.92 & 1.10 \\
\hline
\end{tabular}

Wsp: water sorption, Wsl: water solubility

Table 2: Surface free energy results.

\begin{tabular}{|l|c|c|c|c|}
\hline Group & $\begin{array}{c}\text { Minimum SFE } \\
\left(\mathbf{m} J / \mathbf{m}^{2}\right)\end{array}$ & $\begin{array}{c}\text { Maximum } \\
\text { SFE }\left(\mathbf{m} / \mathbf{m}^{2}\right)\end{array}$ & $\begin{array}{c}\text { Median SFE } \\
\left(\mathbf{m} \mathbf{J} / \mathbf{m}^{2}\right)\end{array}$ & $\begin{array}{c}\text { Interquartile } \\
\text { range }\end{array}$ \\
\hline $\begin{array}{l}\text { Uncoated } \\
\text { PMMA }\end{array}$ & 22.74 & 78.33 & 38.36 & 22.40 \\
\hline $\begin{array}{l}\text { Parylene-coated } \\
\text { PMMA }\end{array}$ & 11.74 & 27.78 & 14.52 & 5.04 \\
\hline
\end{tabular}

SFE: Surface free energy

Table 3: Colour differences $(\Delta E)$ of uncoated and Parylene-C coated PMMA specimens after one week immersion in distilled water or coffee.

\begin{tabular}{|c|c|c|c|c|}
\hline Group & $\mathbf{N}$ & $\Delta \mathbf{E}$ Median & $\Delta \mathbf{E}$ minimum & $\Delta$ E maximum \\
\hline PMMA water & 12 & 1.94 & 0.76 & 2.97 \\
\hline Coated-PMMA water & 12 & 2.73 & 1.19 & 9.16 \\
\hline PMMA coffee & 12 & 2.37 & .87 & 5.41 \\
\hline Coated-PMMA coffee & 12 & 2.84 & 0.93 & 8.29 \\
\hline
\end{tabular}


Citation: Motevasselian F, Cheung E, Soo S, Petrie A, Petridis H. The Effect of Parylene-C Coating on the Surface Free Energy, Water Sorption, Solubility and Staining of PMMA. J Chem Applications. 2014;1(1): 7.

before (L0) and after (L1) immersion in each of the two media, were compared. The median, minimum and maximum lightness of the samples before (L0) and after immersion (L1) in different media are presented in Table 4. Mann-Whitney tests revealed that Parylene-C coated samples were significantly lighter on average than PMMA samples $(\mathrm{P}<0.001)$ both before (L0) and after soaking (L1) in the storage media. Also, the effect of staining test on the lightness shift of the two groups was evaluated. Wilcoxon signed ranks test was used to compare the lightness of the specimens before (L0) and after (L1) soaking in each of the two storage media. It was found that there was a statistically significant reduction in $\mathrm{L}^{*}$ value of the PMMA specimens after soaking in water and coffee solution $(\mathrm{P}=0.005$ and 0.004 respectively). However, there was no statistically significant change in the lightness of Parylene-C coated PMMA samples after storage in either of the two media $(\mathrm{P}=0.099$ and 0.388 for water and coffee, respectively). In addition, L1 values of the PMMA specimens which were immersed in water and coffee were compared by MannWhitney statistical analysis. It was found that there was significant difference in the lightness of the PMMA specimens soaked in two media with higher $\mathrm{L}$ values for those soaked in water $(\mathrm{P}=0.009)$. Some pictures of samples are depicted in Figure 2.

\section{SEM photographs}

The images of PMMA and Parylene-C coated specimens before

Table 4: Lightness values of the test materials before and after immersion in water or in coffee.

\begin{tabular}{|c|c|c|c|c|}
\hline Group & & LO & L1 & $P$ value \\
\hline PMMA water & $\begin{array}{c}\text { Median } \\
\text { Minimum } \\
\text { Maximum }\end{array}$ & $\begin{array}{l}58.18 \\
55.72 \\
60.45\end{array}$ & $\begin{array}{l}56.55 \\
54.94 \\
60.78\end{array}$ & 0.005 \\
\hline $\begin{array}{l}\text { Coated } \\
\text { PMMA water }\end{array}$ & $\begin{array}{c}\text { Median } \\
\text { Minimum } \\
\text { Maximum }\end{array}$ & $\begin{array}{l}64.75 \\
62.04 \\
67.58\end{array}$ & $\begin{array}{l}66.72 \\
63.44 \\
70.47\end{array}$ & .099 \\
\hline PMMA coffee & $\begin{array}{c}\text { Median } \\
\text { Minimum } \\
\text { Maximum }\end{array}$ & $\begin{array}{l}57.19 \\
55.14 \\
59.96\end{array}$ & $\begin{array}{l}53.77 \\
51.58 \\
59.90 \\
\end{array}$ & 0.004 \\
\hline $\begin{array}{l}\text { Coated } \\
\text { PMMA coffee }\end{array}$ & $\begin{array}{c}\text { Median } \\
\text { Minimum } \\
\text { Maximum }\end{array}$ & $\begin{array}{l}66.32 \\
62.74 \\
68.81\end{array}$ & $\begin{array}{l}65.63 \\
63.03 \\
71.90\end{array}$ & 0.388 \\
\hline
\end{tabular}

L0: lightness value before soaking, L1: lightness value after soaking

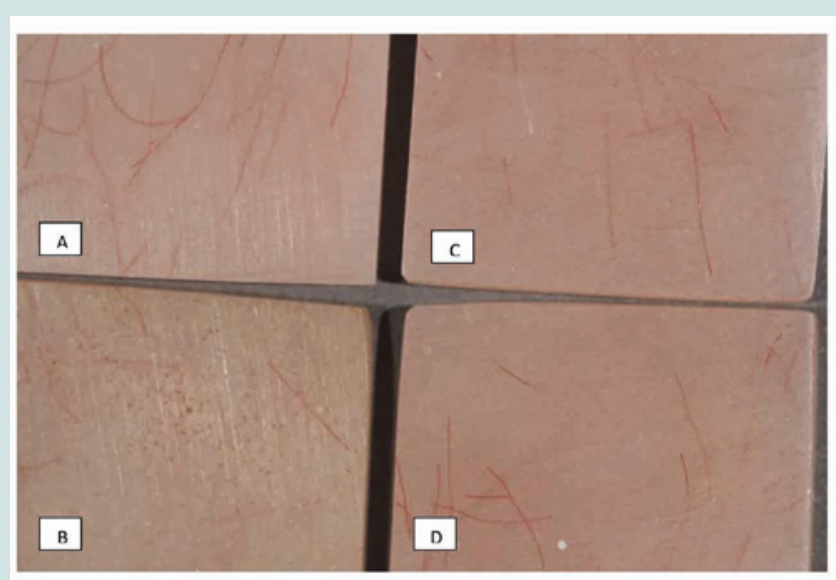

Figure 2: Digital image of different samples after one week immersion in the storage mediums: A) upper left: PMMA and water B) lower left: PMMA and coffee c)upper right: Parylene-coated PMMA and water d) lower right: Parylene-coated PMMA and coffee.
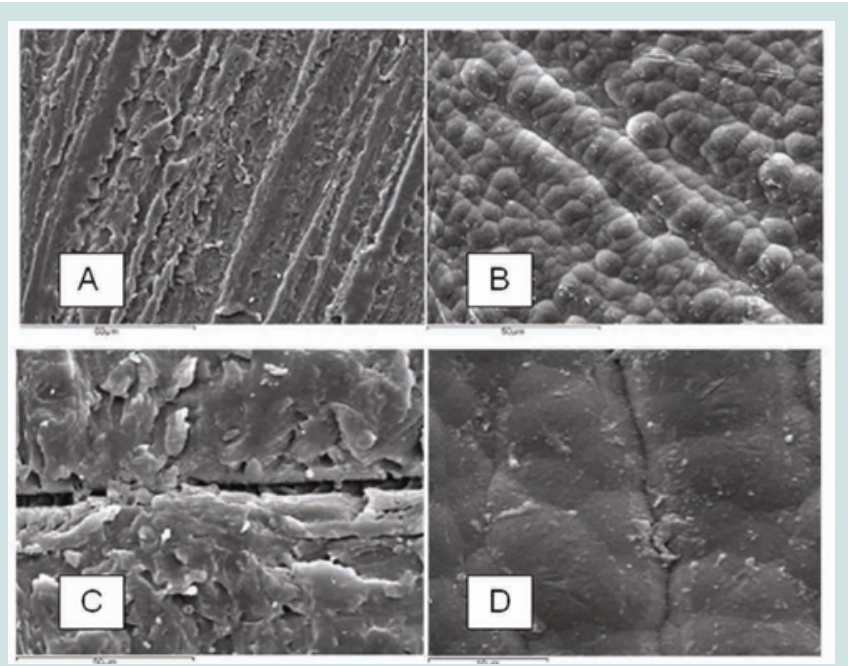

Figure 3: SEM images of uncoated ( $\mathrm{A}$ and $\mathrm{C}$ ) and a Parylene-coated ( $\mathrm{B}$ and D) PMMA specimens at $\times 1000$ and $X 3500$ magnification respectively.

immersion in the test solutions are presented in Figure 3. From the pictures it was evident that the non-coated PMMA specimen revealed a rougher surface after polishing with silicon sandpaper relative to the surface texture of Parylene-C coated PMMA where the grooves and scratches had been covered by uniform layers of spherical shaped Parylene-C particles.

\section{Discussion}

Various fixed and removable intra-oral prostheses are fabricated using PMMA. However, the material presents with certain inherent shortcomings while in service in the demanding oral environment, such as increased sorption, discoloration, deterioration of mechanical properties, and accumulation of microbial biofilms [1,2,10]. The present study was designed to investigate the effect of surface coating of PMMA, with a biomedical coating polymer, on water sorption, solubility, surface free energy, and staining.

\section{Water sorption}

Water sorption of a material represents the amount of water adsorbed on the surface, and absorbed into the body of the material [29]. It is important because it causes plasticization, lowering of the glass transition temperature ( $\mathrm{Tg}$ ) and may affect the mechanical properties and dimensional stability [30]. It also subjects the material to internal stresses that may result in crack formation and, eventually, fractures [31]. Based on the results of this study, Parylene-C coated PMMA presented with statistically reduced Wsp compared to uncoated PMMA. All samples presented with Wsp values below the acceptable $32 \mu \mathrm{g} / \mathrm{mm}^{3}$ [22]. No other study has looked at the effect of coating applications on PMMA water sorption. The difference in water sorption may be explained by the surface condition of the specimens. Although water sorption always occurs in acrylic resins, the sorption rate is accelerated in rougher materials, which are considered more hydrophilic $[32,33]$. In the present study, the SEM pictures revealed a rougher surface of the uncoated, polished PMMA, relative to the surface texture of Parylene-C coated PMMA. This initial rougher surface may have increased hydrophilicity, leading to increased wetting and water sorption. A recent study [34] investigated 
Citation: Motevasselian F, Cheung E, Soo S, Petrie A, Petridis H. The Effect of Parylene-C Coating on the Surface Free Energy, Water Sorption, Solubility and Staining of PMMA. J Chem Applications. 2014;1(1): 7.

the effect of Parylene coating on the surface roughness of PMMA after subjected to cycles of brushing. The results showed that the Parylene coating maintained a statistically significant reduced surface roughness [34].

Another possible explanation for the reduced rate of Wsp of the coated specimens could be the fact that the coating itself was hydrophobic [20]. This was evident by the statistically significant reduction in surface free energy that occurred on coated samples.

\section{Water solubility}

The solubility of the acrylic resins that was measured represented the amount of water soluble ingredients, unreacted monomers, plasticizers, and initiators that leached out during the seven days the specimens were immersed in water [35]. This is important to investigate as these by-products have the potential to induce allergies [36] and cytotoxicity $[37,38]$. The results of this study indicated that the median values of Wsl of both groups were below the requirement of $1.6 \mu \mathrm{g} / \mathrm{mm}^{3}$ [22]. The median values of water solubility for PMMA and Parylene-C coated PMMA were 1.06 and $0.97 \mu \mathrm{g} / \mathrm{mm}^{3}$ respectively and were not significantly different. This suggested that the coating did not act as an effective barrier for reducing the leakage of water soluble components. There is no clear explanation regarding the effect of the Parylene coating on Wsl. The surface condition of a denture plays a significant role in the release of residual monomer [38]. The effect of surface treatment of PMMA by polishing or by coating with another resin on the release of MMA has been investigated [38,39]. Vallittu [38] demonstrated that the polishing of the surface of PMMA reduced the amount of MMA release. This study [38] was based on the hypothesis that the reduced diffusion surface of the specimen brought about by polishing, decreased the MMA release into water. Another study [39] demonstrated that lightcured resin coatings on the surface of PMMA provided an effective barrier against residual MMA release into surrounding water environment for 48 hours. The results of the present study showed that Parylene- $C$ coating did not result in any significant reduction in the release of water soluble materials from PMMA during seven days water immersion, in spite of the smoother surface compared to untreated PMMA samples [34]. It may be suggested that Parylene-C film does not provide a highly cross-linked cover on the surface to suppress the leaching of the water soluble ingredients. Barrier performance of Parylene $\mathrm{C}$ may be dependent upon its thickness and more research is needed in this direction in order to optimize it for future use. Another factor which might have affected Wsl could be the stability of the adhesion of the coating on the PMMA substrate. One of the limitations of Parylene- $\mathrm{C}$ coatings is their poor adhesion to some substrates. Parylene, through its deposition process, does not adhere chemically, only mechanically [40]. The largest factor affecting Parylene adhesion is surface cleanliness and it is highly recommended that the substrate be cleaned prior to coating [4143]. Moreover, for optimal adhesion of Parylene to a wide variety of substrates, surface treatment with plasma and adhesion promoting agents prior to Parylene coating is performed [41-43], but details of these treatments remain undisclosed. Nevertheless, a previous study [34] demonstrated that Parylene was still adhering to PMMA even after mechanical challenges. More studies should be performed on the stability of the bond to the substrate, especially after artificial aging.

\section{Surface free energy}

Surface free energy is one of the most important physicochemical properties of a material that play a role during the early phases of microbial attachment. Being able to reduce the surface free energy of a material in a clinical situation would directly influence the amounts of initial microbial attachment [44,45]. This may be of particular importance in medicine and dentistry in the case of PMMA, which has been proven to retain such fungal and bacterial biofilms when used for the fabrication of intraoral prostheses. These PMMA biofilms have been repeatedly found to promote both local and, more importantly, systemic, potentially life-threatening infections in susceptible cohorts such as diabetic patients, hospitalised populations and other immune-compromised patients [46].

The results of this study showed that Parylene C coating significantly reduced the surface free energy levels of PMMA in a laboratory setting. This was expected due to the nature of Parylene polymers [47]. The reduced surface free energy levels of the test group could be explained by the fact that Parylene $\mathrm{C}$ has an all-carbon 'backbone' which has no oxygen, nitrogen or sulphur atom links [47]. This chemical makeup is what gives Parylene $\mathrm{C}$ its low surface free energy. A previous study [48] did not find any statistically significant difference in surface free energy between Parylene coated and uncoated PMMA samples. The difference in results may be explained by the fact that in that study [48], the variant Parylene $\mathrm{N}$ was used that is chemically different to Parylene $\mathrm{C}$ used in this study, as well as by the different testing liquids used to calculate SFE.

The results of this study also showed that the interquartile range of the uncoated control group was more than four times greater than the test group $(22.40 \mathrm{mN} / \mathrm{m}$ to $5.04 \mathrm{mN} / \mathrm{m})$. The lower range of values of the coated group may be explained by the effect of the coating on masking-out the various surface irregularities and microdefects of PMMA. As Parylene is highly conformal, it is able to fill in the microscopic pits and troughs of the PMMA surface during the coating process. In doing so, it provides PMMA with a more conformal, uniform and continuous surface that effectively reduces the surface roughness of the material. This surface uniformity increases smoothness which in turn reduces its surface free energy values.

\section{Staining}

The CIE Lab system is widely used in determining colour changes through the evaluation and expression of differences in three dimensions of colour space, thus obtaining mathematical data [1]. The three coordinates of CIE Lab represent the lightness of the colour ( $\mathrm{L}^{*}=0$ yields black and $\mathrm{L}^{*}=100$ indicates diffuse white), its position between $\mathrm{red} / \mathrm{magenta}$ and green $\left(\mathrm{a}^{*}\right.$ negative values indicate green while positive values indicate magenta) and its position between yellow and blue $\left(\mathrm{b}^{*}\right.$ negative values indicate blue and positive values indicate yellow) [28]. Judging the colour of a surface highly depends on the both the actual colours of the surface and the lighting conditions under which the surface is measured [49], time of the day, surrounding conditions and position of the specimen [50,51]. Smoothness of the specimen surface also affects colour measurement [49]. The ideal colour temperature of the light source for colour 
Citation: Motevasselian F, Cheung E, Soo S, Petrie A, Petridis H. The Effect of Parylene-C Coating on the Surface Free Energy, Water Sorption, Solubility and Staining of PMMA. J Chem Applications. 2014;1(1): 7.

reproduction is $5,500 \mathrm{~K}$ [50]. In the present study, since colour differences were being tested under uniform conditions, the choice of the illuminant was not critical [49].

In this study, it was expected that fine colorant particles of coffee solution could have been deposited more readily into pits and grooves of the rougher surface of uncoated PMMA samples compared with the smoother surface of Parylene-C coated ones. However, digital image analysis did not show any significant difference of $\Delta \mathrm{E}$ values between the groups. The coating only seemed to affect $L^{\star}$ values, the results showing that the $L^{*}$ values of the uncoated samples before and after immersion in the test solutions were consistently lower compared to the Parylene-coated samples. As the SEM images showed, the uncoated PMMA samples presented with a rougher surface compared to the coated notes [34]. This roughness may have caused reflection, refraction and scattering of the incident light in different directions resulting in a reduction in value. On the other hand, the spherical Parylene particles formed a smooth and uniform surface on the coated samples which might have resulted in a reflection of the incident light in a more uniform direction, contributing to the higher $\mathrm{L}^{*}$ values measured. This assumption is in agreement with a recent study [52] which evaluated the influence of surface roughness on the colour of dental resin composites and found that lightness increased as roughness decreased. Furthermore, Parylene- $C$ exhibits very little absorption in the visible region and is therefore, colourless in visible light [47]. This property may have led to an increased reflection of incident light, resulting in increased lightness values.

Statistical analysis showed that the CIE Lab values obtained from image analysis of uncoated PMMA specimens decreased significantly after immersion in the test solutions and that the L1 value of the uncoated samples immersed in coffee was less than those in water. This finding is in agreement with other studies $[53,54]$ which showed that staining solutions caused a decrease in lightness. No statistical difference in $L^{*}$ values was seen between the coated sample groups in either solution. This function of the coating may be explained by the fact that Parylene- $\mathrm{C}$ is inert and has very few active sites to form molecular bonds with other chemicals. Buyukyilmaz and Ruyter [13] demonstrated that discoloration of denture materials results from the adhesion of colorants to the surface. Hersek et al. [1] stated that staining might be the result of molecular interactions between colorants and denture polymers. It was also explained that the resin specimens absorb the colorant solution, primarily due to the polar properties of the resin molecules and diffusion mechanism of water molecules. ${ }^{1}$ These properties might explain why the $L^{*}$ values in Parylene-C coated samples were not significantly different in the test solutions, since less adhesion of colorant agents to the inert surface of Parylene-C film may occur.

Further research should be conducted on the application of Parylene coatings on PMMA. Factors such as storage time and temperature may alter Wsp and Wsl values and should be investigated with an emphasis on the possible qualitative differences of the released chemicals between coated and uncoated PMMA. Other factors, such as initial PMMA surface roughness and coating thickness deserve further research. Future studies should also address the direct effect of the coating on biofilm retention. Some of these topics are already under investigation by the authors.

\section{Conclusions}

Within the limits of this study, the following conclusions were drawn:

- Parylene C coated PMMA samples exhibited, on average, lower water sorption and lower surface free energy compared to uncoated samples.

- Parylene C coating did not have a statistically significant effect on water solubility values.

- No significant difference in $\Delta \mathrm{E}$ values was found between all groups of test materials after soaking in distilled water and coffee solution.

- Parylene coated PMMA presented with significantly higher $L^{\star}$ values, which were not affected after soaking in media, compared to uncoated PMMA.

\section{References}

1. Hersek N, Canay S, Uzum G, Yildiz F (1999) Colour stability of denture base resins in three food colorants. J Prosthet Dent 81: 375-379.

2. Imirzalioglu P, Karacaer O, Yilmaz B, Ozmen I (2010) Colour stability of denture acrylic resins and a soft lining material against tea, coffee, and nicotine. J Prosthodont 19: 118-124.

3. Polat TN, Karacaer O, Tezvergil A, Lassila LV, Vallittu PK (2003) Water sorption, solubility and dimensional changes of denture base polymers reinforced with short glass fibers. J Biomater Appl 17: 321-325.

4. Monfrin SB, Notaro V, Gassino G, Perotti R, Bassi F (2005) Dimensional contour stability of acrylic resin bases for complete dentures before and after water sorption. Int J Prosthodont 18: 480-482.

5. Takahashi Y, Chai J, Kawaguchi M (1999) Equilibrium strengths of denture polymers subjected to long-term water immersion. Int J Prosthodont 12: 348352.

6. Takahashi Y, Hamanaka I, Shimizu H (2012) Effect of thermal shock on mechanical properties of injection-molded thermoplastic denture base resins. Acta Odontol Scand 70: 297-302.

7. Wady AF, Machado AL, Vergani CE, Pavarina AN, Giampaolo ET (2011) Impact strength of denture base and reline acrylic resins subjected to long term water immersion. Brazil Dent J 22: 56-61.

8. Vallittu PK, Ruyter IE, Ekstrand K (1998) Effect of water storage on the flexural properties of E-glass and silica fiber acrylic resin composite. Int $\mathrm{J}$ Prosthodont 11: 340-350.

9. Chen CC, Shen J, Saver JA (1985) Effects of sorbed water on properties of low and high molecular weight PMMA: 11 . Fatigue performance. Polymer 26 : 89-96.

10. Anusavice KJ (2003) Phillips' Science of dental material. (11thedn), Saunders, Philadelphia.

11. Moura JS, da Silva WJ, Pereira T, Del Bel Cury AA, Rodrigues Garcia RC (2006) Influence of acrylic resin polymerization methods and saliva on the adherence of four Candida species. J Prosthet Dent 96: 205-211.

12. Zissis AJ, Polyzois GL, Yannikakis SA, Harrison A (2000) Roughness of denture materials: a comparative study. Int J Prosthodont 13: 136-140.

13. Buyukyilmaz S, Ruyter IE (1994) Colour stability of denture base polymers. Int J Prosthodont 7: 372-382.

14. Hong G, Murata H, Li Y, Sadamori S, Hamada T (2009) Influence of denture cleansers on the color stability of three types of denture base acrylic resin. J Prosthet Dent 101: 205-213.

15. Sepúlveda-Navarro WF, Arana-Correa BE, Borges CP, Jorge JH, Urban VM et al. (2011) Color stability of resins and nylon as denture base material in beverages. J Prosthodont 20: 632-638. 
Citation: Motevasselian F, Cheung E, Soo S, Petrie A, Petridis H. The Effect of Parylene-C Coating on the Surface Free Energy, Water Sorption, Solubility and Staining of PMMA. J Chem Applications. 2014;1(1): 7.

16. Song JS, Lee S, Jung SH, Cha GC, Mun MS (2009) Improved biocompatibility of Parylene-C films prepared by chemical vapour deposition and the subsequent plasma treatment. J Appl Poly Sci 112: 3677-3685.

17. Loeb GE, Walker AE, Uematsu S, Konigsmark BW (1977) Histologica reaction to various conductive and dielectric films chronically implanted in the subdural space. J Biomed Mater Res 11: 195-210.

18. Chang TY, Yadav VG, De Leo S, Mohedas A, Rajalingam B, et al. (2007) Cell and protein compatibility of parylene-C surfaces. Langmuir 23: 11718-11725.

19. Hahn AW, Yasuda HK, James WJ, Nichols MF, Sadhir RK, et al. (1981) Glow discharge polymers as coatings for implanted devices. Biomed Sci Instrum 17: 109-113.

20. Tooker A, Meng E, Erickson J, Tai YC, Pine J (2005) Biocompatible parylene neurocages. Developing a robust method for live neural network studies. IEEE Eng Med Biol Mag 24: 30-33.

21. Zhou L, Tong Z, Wu G, Feng Z, Bai S, et al. (2010) Parylene coating hinders Candida Albicans adhesion to silicone elastomers and denture bases resin. Arch Oral Biol 55:401-409.

22. European Standard. Dentistry-Base polymers (2008) EN ISO 20795-1.

23. Owens DK, Wendt RC (1969) Estimation of surface free energy of polymers J Appl Polym Sci 13: 1741-1747.

24. Clydesdale FM (1978) Colorimetry--methodology and applications. CRC Crit Rev Food Sci Nutr 10: 243-301

25. Freeman M (2011) The exposure field guide. The essential handbook to getting the perfect exposure in photography; any subject, anywhere. (1stedn), Focal Press, Burlington.

26. Kim SH, Lee YK (2009) Measurement of discolouration of orthodontic elastomeric modules with a digital camera. Eur J Orthod 31: 556-562.

27. Yam KL, Papadakis SE (2004) A simple digital imaging method for measuring and analyzing colour of food surfaces. J Food Eng 61: 137-142.

28. Commission Internationale de I' Eclairge (CIE) (2004) (3rdedn) Bureau Central de La Cle, Vienna. CIE publication no.15.

29. Craig RG, Powers JM (2002) Restorative dental materials (11thedn), Mosby, St. Louis.

30. Tham WL, Chow WS, Mohd Ishak ZA (2010) Simulated body fluid and water absorption effects on poly(methyl methacrylate)/hydroxyapatite denture base composites. eXPRESS Polymer Letters 4: 517-528.

31. Hiromori K, Fujii K, Inoue K (2000) Viscoelastic properties of denture base resins obtained by underwater test. J Oral Rehabil 27: 522-531.

32. Monsénégo P, Baszkin A, Costa ML, Lejoyeux J (1989) Complete denture retention, Part II: wettability studies on various acrylic resin denture base materials. J Prosthet Dent 62: 308-312.

33. Kilanim BH, Retief DH, Guldag MV, Castleberry DJ, Fischer TE (1984) Wettability of selected denture base materials. J Prosthet Dent 52: 288-291.

34. Santos M, Soo S, Petridis H (2013) The effect of Parylene coating on the surface roughness of PMMA after brushing. J Dent 41:802-808.

35. Cucci AL, Vergani CE, Giampaolo ET, Afonso MC (1998) Water sorption, solubility, and bond strength of two auto-polymerizing acrylic resins and one heat-polymerizing acrylic resin. J Prosthet Dent 80: 434-438.

36. Lygre H (2002) Prosthodontic biomaterials and adverse reactions: a critical review of the clinical and research literature. Acta Odontol Scand 60: 1-9.

37. Jorge JH, Giampaolo ET, Machado AL, Vergani CE (2003) Cytotoxicity of denture base acrylic resins: a literature review. J Prosthert Dent 90: 190-193.

38. Vallittu PK (1996) The effect of surface treatment of denture acrylic resin on the residual monomer content and its release into water. Acta Odontol Scand 54: 188-192.

39. Tsuchiya H, Yamada K, Akagiri M, Tajima K, Miyazaki T, et al. (1993) Effect of an ultraviolet light-activated coating material on reduction of the leaching of methyl methacrylate and formaldehyde from denture acrylic resins. Dent Mater J 12: 253-258.
40. Khabari A, Urban FK (2005) Partially ionized beam deposition of parylene. J Non Cryst Solids 351: 3536-3541.

41. Hwang KS, Park JH, Lee JH, Yoon DS, Kim TS, et al. (2004) Effect of Atmospheric-Plasma Treatments for Enhancing Adhesion of Au on Parylenec-Coated Protein Chips. J Korean Phys Soc 44: 1168-1172.

42. Lee JH, Hwang KS, Kim TS (2004) Effect of Oxygen Plasma Treatment on Adhesion Improvement of Au Deposited on Pa-c Substrates. J Korean Physical Soc 44: 1177-1181.

43. Zeniieh D, Bajwa A, Ledernez L, Urban G (2013) Effect of Plasma Treatments and Plasma-Polymerized Films on the Adhesion of Parylene-C to Substrates. Plasma Processes and Polymers 10: 1081-1089.

44. Liu Y, Zhao Q (2005) Influence of surface energy of modified surfaces on bacterial adhesion. Biophys Chem 117: 39-45.

45. Quirynen M, Van Der Mei HC, Bollen CM, Van Der Bossche LH, Doornbusch GI, et al. (1994) Clinical relevance of the influence of surface free energy and roughness on the supragingival and subgingival plaque formation in man. Colloids Surf B: Biointerfaces 2: 25-31.

46. Smith AJ, Jackson MS, Bagg J (2001) The ecology of Staphylococcus species in the oral cavity. J Med Microbiol 50: 940-946.

47. Kumar R (2008) Xylene polymers. Kirk-Othmer Encyclopedia of Chemical Technology. John Wiley and Sons Inc. 1-52.

48. Zhou L, Tong Z, Wu G, Feng Z, Bai S, et al. (2010) Parylene coating hinders Candida Albicans adhesion to silicone elastomers and denture bases. Arch Oral Biol 55: 401-409

49. Guler AU, Yilmaz F, Kulunk T, Guler E, Kurt S (2005) Effects of different drinks on stain ability of resin composite provisional restorative materials. J Prosthet Dent 94: 118-124.

50. Fondriest J (2003) Shade matching in restorative dentistry: the science and strategies. Int J Periodont Rest Dent 23: 467-479.

51. Joiner A (2004) Tooth colour: a review of the literature. J Dent 32: 3-12.

52. Ghinea R, Ugarte-Alvan L, Yebra A, Pecho OE, Paravina RD, et al. (2011) Influence of surface roughness on the colour of dental-resin composites, $J$ Zhejiang Univ Sci B 12: 552-562.

53. Lath DL, Johnson C, Smith RN, Brook AH (2006) Measurement of stain removal in vitro: a comparison of two instrumental methods. Int J Dent Hyg 4: 129-132.

54. Lath DL, Smith RN, Guan YH, Karmo M, Brook AH (2007) Measurement of stain on extracted teeth using spectrophotometry and digital image analysis. Int J Dent Hyg 5: 174-179.

\section{Acknowledgements}

Mr Aaron Clark (SCS Coatings, Surrey, England) for the kind offer to coat the samples Mr Colin Clark, for his technical advice (Eastman Dental Institute, UCL). 